\title{
Oligodendrocyte abnormalities in layer 5 in the inferior parietal lobule are associated with lack of insight in schizophrenia: A postmortem morphometric study
}

\author{
Natalya A. Uranova* \\ Victor M. Vostrikov \\ Natalya S. Kolomeets \\ Laboratory of Clinical Neuropathology, \\ Mental Health Research Center, \\ Zagorodnoe shosse 2, 117152. \\ Moscow
}

RUSSIA

\begin{abstract}
Background and Objectives: Previously we reported in layer 3 of the inferior parietal lobule a reduction in the numerical density of oligodendrocytes ( $\mathrm{Nv} \mathrm{O}$ ), oligodendrocyte clusters (Nv O1C) in BA 39, and in the number of perineuronal oligodendrocytes (N PnOl) in BA 39/40 areas in schizophrenia. These changes were associated with lack of insight. We hypothesized that similar abnormalities might occur in layer 5 in BA 39/40, and they might be associated with lack of insight.

Methods: We estimated the Nv Ol, the Nv OlC by optical disector method and the N $\mathrm{PnOl}$ in layer 5 in BA 39/40 in Nissl stained sections from 24 males with schizophrenia and 24 normal male controls from the Stanley Parietal Collection. The schizophrenia group was divided into three subgroups based on level of insight: poor, fair or good.

Results: We found a significant deficit in the parameters measured in BA 39 in the schizophrenia group and in the subgroup of subjects having poor insight as compared to the control group. In BA 40 the $\mathrm{Nv} \mathrm{Ol}$ and the $\mathrm{Nv} \mathrm{OlC}$ were significantly lower in the schizophrenia group compared to controls, and the $\mathrm{N} \mathrm{PnOl} \mathrm{was} \mathrm{not} \mathrm{changed.} \mathrm{Each} \mathrm{insight}$ subgroup showed a decreased the $\mathrm{Nv} \mathrm{Ol}$ and the $\mathrm{Nv}$ OlC compared to controls. There were no subgroup differences in BA 39/40.

Conclusions: Schizophrenia is characterized by the reduction in the Nv Ol and the Nv OlC in layer 5 of BA 39/40. Oligodendrocyte abnormalities in BA 39 are associated with poor insight in schizophrenia.
\end{abstract}

Received: 28 April 2015

Revised: 17 May 2015

Accepted: 17 Juny 2015 


\section{Introduction}

The inferior parietal lobule (IPL) consists of the supramarginal gyrus (BA 40) and adjacent angular gyrus (BA 39). The IPL participates in sensory integration, body image, concept of self and in executive functions ${ }^{1}$. Impaired insight is a common feature in psychosis, it predicts outcome, prognosis and treatment adherence. Lack of insight is associated with structural and functional brain abnormalities. Lower grey matter volumes in the temporal and parietal regions are associated with poor insight in psychosis ${ }^{2}$, and IPL activity was correlated with cognitive insight in schizophrenia patients ${ }^{3}$. Decreased activity of the IPL has been recently reported in schizophrenia patients ${ }^{3-5}$.

Previously we reported a significant reduction in the numerical density of oligodendrocytes $(\mathrm{Nv} \mathrm{Ol})^{6}$ and oligodendrocyte clusters $(\mathrm{Nv} \mathrm{OlC})^{7}$ in layer 3 in BA 39 in the IPL in schizophrenia. We also found a deficit of perineuronal oligodendrocytes $(\mathrm{N} \mathrm{PnOl})$ adjacent to pyramidal neurons in layer 3 in BA 39 and BA 40 in schizophrenia ${ }^{8}$. These changes were associated with lack of insight ${ }^{6-8}$. Deficit of OlC was also found in BA 40 in schizophrenia $^{7}$. Besides, a significant lateralization of oligodendrocyte density was detected in layer 3 (L>R) in BA 39 only in the control group ${ }^{6-8}$. We hypothesized that similar oligodendrocyte abnormalities might occur in layer 5 in BA 39/40, and they might be associated with lack of insight.

The aim of the present study was to estimate the Nv Ol, the Nv OlC and N PnOl adjacent to pyramidal neurons in layer 5 in the same BA 39/40 areas previously studied in schizophrenia and controls to detect the effects of diagnosis, hemispheres and insight.

\section{Materials and methods}

\section{Samples}

Human brain specimens were donated by the Stanley Medical Research Institute's 'Parietal Collection'. The samples consisted of 48 male subjects ( 24 controls and 24 with schizophrenia). Diagnosis was made according to DSM-IV criteria. A postmortem estimate of each person's awareness of illness (insight) based on the person's clinical records has been previously reported ${ }^{6}$. Complete demographic and clinical data were reported in the previous paper ${ }^{6}$.

The brain specimens were coded, and all cytoarchitectural assessments were done blindly. The angular gyrus (BA 39) and the supramarginal gyrus (BA 40) were identified according to macroscopic landmarks ${ }^{12}$. Ten serial sections through the IPL (every 17th section) were mounted on slides and Nissl-stained.

\section{Morphometric analysis}

The layer 5 in BA 39 and in BA 40 was easily identified based on cytoarchitectural features. The $\mathrm{Nv} \mathrm{Ol}$ and the $\mathrm{Nv} \mathrm{OlC}$ were estimated by optical disector method as previously described ${ }^{6,7}$. To estimate the Nv Ol, the Nv OlC and the N PnOl 100 fields were counted for each parameter per case. Oligodendrocytes were identified by the presence of a small round or oval nucleus, with relatively dense nuclear staining (more chromophilic than astroglial nuclei) and a narrow unstained rim of cytoplasm. PnO1 were defined as all oligodendrocytes located within $<5 \mu \mathrm{m}$ of pyramidal neurons. Pyramidal neurons were identified by their triangular shape, oval shaped clear nucleus with a prominent nucleolus, presence of intensively stained cy- 
toplasm and vertical apical dendrite. Pyramidal neurons with identifiable nucleolus were systematically randomly sampled. The number of pyramidal cells sampled from case for each Brodmann's area was 100, from each group 2,400, total 4,800. The $\mathrm{N} \mathrm{PnOl} \mathrm{was} \mathrm{ex-}$ pressed as the number of oligodendrocytes per neuron.

\section{Statistical analysis}

Statistical analysis was performed using Statistica 7. The Kolmogorov-Smirnov test showed normal distribution of the parameters measured. A Pearson correlation analysis was performed to assess possible correlations between the parameters measured and age, postmortem interval, $\mathrm{pH}$, refrigerator interval, brain weight, total lifetime antipsychotics, age at onset and duration of disease. A one-way MANOVA with the $\mathrm{Nv} \mathrm{Ol,} \mathrm{the}$ $\mathrm{Nv} \mathrm{OlC}$ and the $\mathrm{N} \mathrm{PnOl}$ as the dependent variables was used to compare the control group and the schizophrenia group and to compare the control group with the three insight schizophrenia subgroups. To test hemispheric effects a two-way MANOVA with diagnosis and hemispheres as the independent variables was performed. The analyses were followed by post hoc Duncan's test.

\section{Results}

\section{Brodmann's area 39}

The mean Nv Ol, Nv OlC, $\mathrm{N} \mathrm{PnOl}$ and the results of their comparisons between the schizophrenia and the control groups are given in Table 1. A significant reduction in the mean values of the $\mathrm{Nv} \mathrm{Ol} \mathrm{(19 \% ),} \mathrm{the} \mathrm{Nv}$ OlC $(-19 \%)$ and the $\mathrm{N}$ PnOl $(-16 \%)$ were found in the schizophrenia group as compared to the control group.

Table 1

The numerical density of oligodendrocytes ( $\mathrm{Nv} \mathrm{Ol}$ ), oligodendrocyte clusters ( $\mathrm{Nv} \mathrm{OlCl),} \mathrm{and} \mathrm{of} \mathrm{the} \mathrm{number}$ of perineuronal oligodendrocytes $(\mathrm{N} \mathrm{PnOl})$ (means $\pm \mathrm{SD})$, effect of diagnosis, BA 39.

\begin{tabular}{lcccc} 
Parameters & Controls $(\mathrm{n}=24)$ & Schizophrenia $(\mathrm{n}=24)$ & $\mathrm{F}(1,46)$ & $\mathrm{p}$ \\
\hline Nv Ol & $127.82 \pm 31.0$ & $104.07 \pm 25.91$ & 8.29 & 0.006 \\
Nv OlC & $22.59 \pm 6.39$ & $18.44 \pm 5.28$ & 6.00 & 0.020 \\
N PnOl & $1.55 \pm 0.30$ & $1.30 \pm 0.24$ & 9.69 & 0.003 \\
\hline
\end{tabular}

There were no significant hemispheric differences in each parameter measured (all $p>0.2$ for the control and the schizophrenia groups).

We found a significant effect of insight on these parameters (Table 2). The subgroups of subjects having poor insight and fair insight had lower mean values in the $\mathrm{Nv} \mathrm{Ol}(-24 \%$,
$-23 \%$ respectively, $\mathrm{p}<0.05)$ as compared to the control group. Only the subgroup having poor insight had significantly lower the $\mathrm{Nv}$ OlC $(-28 \%, \mathrm{p}<0.05)$ and the $\mathrm{N} \mathrm{PnOl}(-20 \%$, $\mathrm{p}<0.05)$ as compared to the control group. The subjects with good insight did not differ from the subjects with poor insight. 
Table 2

The numerical density of oligodendrocytes (Nv Ol), oligodendrocyte clusters (Nv OlC), and of the number of perineuronal oligodendrocytes ( $\mathrm{N} \mathrm{PnOl}$ ) (means $\pm \mathrm{SD}$ ), effect of insight, BA 39.

\begin{tabular}{lcccccc} 
Parameters & $\begin{array}{c}\text { Controls } \\
(\mathrm{n}=24)\end{array}$ & $\begin{array}{c}\text { Poor insight } \\
(\mathrm{n}=10)\end{array}$ & $\begin{array}{c}\text { Fair insight } \\
(\mathrm{n}=5)\end{array}$ & $\begin{array}{c}\text { Good insight } \\
(\mathrm{n}=9)\end{array}$ & $\mathrm{F}(3,44)$ & $\mathrm{p}$ \\
\hline $\mathrm{Nv}$ Ol & $127.82 \pm 31.00$ & $97.57 \pm 25.08$ & $98.43 \pm 30.57$ & $114.43 \pm 27.70$ & 3.39 & 0.03 \\
$\mathrm{Nv}$ OlC & $22.59 \pm 6.39$ & $16.29 \pm 4.66$ & $17.20 \pm 5.91$ & $21.59 \pm 4.47$ & 3.60 & 0.02 \\
$\mathrm{~N} \mathrm{PnOl}$ & $1.55 \pm 0.30$ & $1.24 \pm 0.20$ & $1.28 \pm 0.15$ & $1.37 \pm 0.33$ & 3.53 & 0.02 \\
\hline
\end{tabular}

\section{Brodmann's area 40}

The mean Nv Ol, the Nv OlC and the $\mathrm{N}$ $\mathrm{PnOl}$ and the results of their comparisons between the schizophrenia and the control groups are given in Table 3.

There was a significant reduction in the mean values of the $\mathrm{Nv} \mathrm{Ol}(-28 \%)$, the $\mathrm{Nv}$ $\mathrm{OlC}(-42 \%)$ and a trend to the reduction in the $\mathrm{N} \mathrm{PnOl}(-14 \%)$ in the schizophrenia group as compared to the control group.
There were no significant hemispheric differences in each parameter measured (all $\mathrm{p}>$ 0.4 for the control and the schizophrenia groups).

The results of one-way ANOVA comparison of the control group with the three insight subgroups are given in Table 4. Three insight subgroups differed from the control group in the $\mathrm{Nv} \mathrm{Ol}$ and in the $\mathrm{Nv} \mathrm{OlC}$. None of insight subgroups differed from the control group in the N Pn Ol. In contrast to BA 39 in

\section{Table 3}

The numerical density of oligodendrocytes ( $\mathrm{Nv} \mathrm{O1}$ ), oligodendrocyte clusters (Nv OlC), and of the number of perineuronal oligodendrocytes $(\mathrm{N} \mathrm{PnOl})($ means $\pm \mathrm{SD})$, effect of diagnosis, BA 40.

\begin{tabular}{lcccc} 
Parameters & Controls $(\mathrm{n}=24)$ & Schizophrenia $(\mathrm{n}=24)$ & $\mathrm{F}(1,46)$ & $\mathrm{p}$ \\
\hline Nv Ol & $142.05 \pm 26.05$ & $102.74 \pm 23.74$ & 29.8 & $<0.001$ \\
Nv OlC & $24.05 \pm 6.06$ & $14.1 \pm 4.42$ & 42.3 & $<0.001$ \\
N PnOl & $1.12 \pm 0.29$ & $0.97 \pm 0.24$ & 3.6 & 0.06 \\
\hline
\end{tabular}

Table 4

The numerical density of oligodendrocytes ( $\mathrm{Nv} \mathrm{Ol}$ ), oligodendrocyte clusters (Nv OlC), and of the number of perineuronal oligodendrocytes (N PnOl) (means \pm SD), effect of insight, BA 40.

\begin{tabular}{lcccccc} 
Parameters & $\begin{array}{c}\text { Controls } \\
(\mathrm{n}=24)\end{array}$ & $\begin{array}{c}\text { Poor insight } \\
(\mathrm{n}=10)\end{array}$ & $\begin{array}{c}\text { Fair insight } \\
(\mathrm{n}=5)\end{array}$ & $\begin{array}{c}\text { Good insight } \\
(\mathrm{n}=9)\end{array}$ & $\mathrm{F}(3,44)$ & $\mathrm{p}$ \\
\hline $\mathrm{Nv} \mathrm{Ol}$ & $142.16 \pm 26.05$ & $102.93 \pm 24.2$ & $89.11 \pm 26.73$ & $110.11 \pm 20.67$ & 10.78 & $<0.001$ \\
$\mathrm{Nv}$ OlC & $24.05 \pm 6.06$ & $13.64 \pm 4.28$ & $11.36 \pm 2.98$ & $16.12 \pm 4.64$ & 15.25 & $<0.001$ \\
$\mathrm{~N} \mathrm{PnOl}$ & $1.12 \pm 0.29$ & $0.95 \pm 0.26$ & $1.0 \pm 0.3$ & $0.98 \pm 0.19$ & 1.2 & 0.32 \\
\hline
\end{tabular}




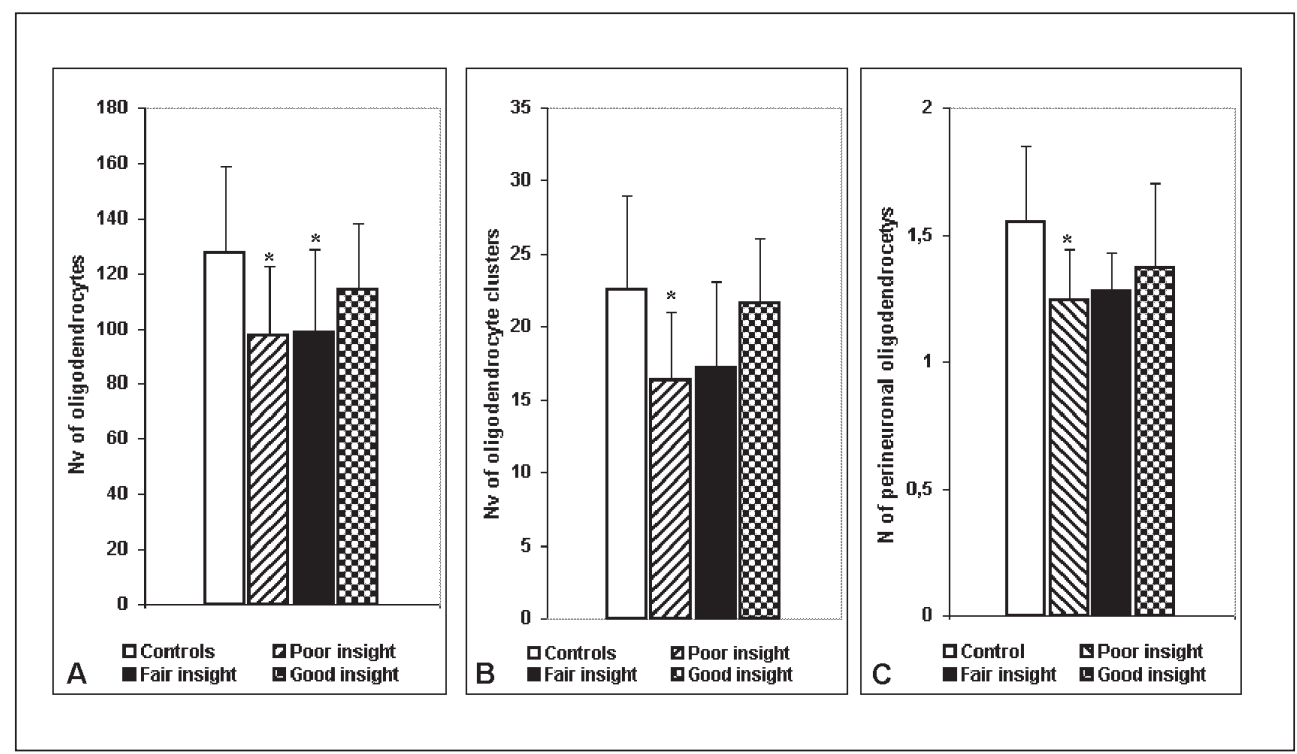

Figure 1. The numerical density of oligodendrocytes ( $\mathrm{Nv} \mathrm{Ol}$ ( $(\mathrm{A})$, oligodendrocyte clusters (Nv OlC) (B) and the number of perineuronal oligodendrocytes $(\mathrm{N} \mathrm{PnOl})(\mathrm{C})$ in $\mathrm{BA} 39$ in the control group and in different insight subgroups. Data are given as mean \pm standard deviation. p-values: $* \mathrm{p}<0.05 ; * * \mathrm{p}<0.01$

BA 40 each of three insights subgroups differed from the control group. The subjects having poor insight had significantly lower mean values in the $\mathrm{Nv} \mathrm{Ol}(-28 \%, \mathrm{p}<0.001)$ and in the Nv of OlC $(-44 \%, \mathrm{p}<0.001)$ as compared to controls. The subjects having fair insight had significantly lower mean values in the $\mathrm{Nv} \mathrm{Ol}(-38 \%, \mathrm{p}<0.001)$ and in the $\mathrm{Nv}$ OlC $(-53 \%, \mathrm{p}<0.001)$ as compared to controls. The subjects having good insight also had a significantly lower mean values in the $\mathrm{Nv} \mathrm{Ol}(-33 \%, \mathrm{p}<0.001)$ and in the $\mathrm{Nv}$ of OlC $(-33 \%, \mathrm{p}<0.001)$ as compared to the control group. There were no significant differences between the subgroups (Fig. 2).

\section{Potential confounding factors}

Correlation analysis did not revealed any interactions between the parameter measured and age, postmortem interval, refrigerator interval, brain weight, brain $\mathrm{pH}$, lifetime antipsychotics in both areas studied.

\section{Discussion}

There are three main results of this study. First, we demonstrated for the first time that the $\mathrm{Nv} \mathrm{Ol}$ and the $\mathrm{Nv} \mathrm{OlC}$ in layer 5 of BA $39 / 40$ areas in schizophrenia were lower as compared to controls. Second, the reduction in the $\mathrm{Nv} \mathrm{Ol}$, in the $\mathrm{Nv} \mathrm{OlC}$ and $\mathrm{N} \mathrm{PnOl}$ in BA 39 were associated with poor insight in schizophrenia. And third, there were no significant hemispheric differences in either control or schizophrenia groups in BA 39/40 areas.

The present study is a continuation of our previous study using the same IPL section collection. Previously we reported a significant reduction in the $\mathrm{Nv} \mathrm{Ol}(-15 \%)$ and in the $\mathrm{Nv}$ OlC $(-23 \%)$ in layer 3 in BA 39 in schizophrenia as compared to normal controls ${ }^{6,7}$. We also found a deficit of $\mathrm{N} \mathrm{PnOl}$ adjacent to pyramidal neurons in layer 3 in BA 39 (-20\%) as compared to the controls ${ }^{8}$. These changes 


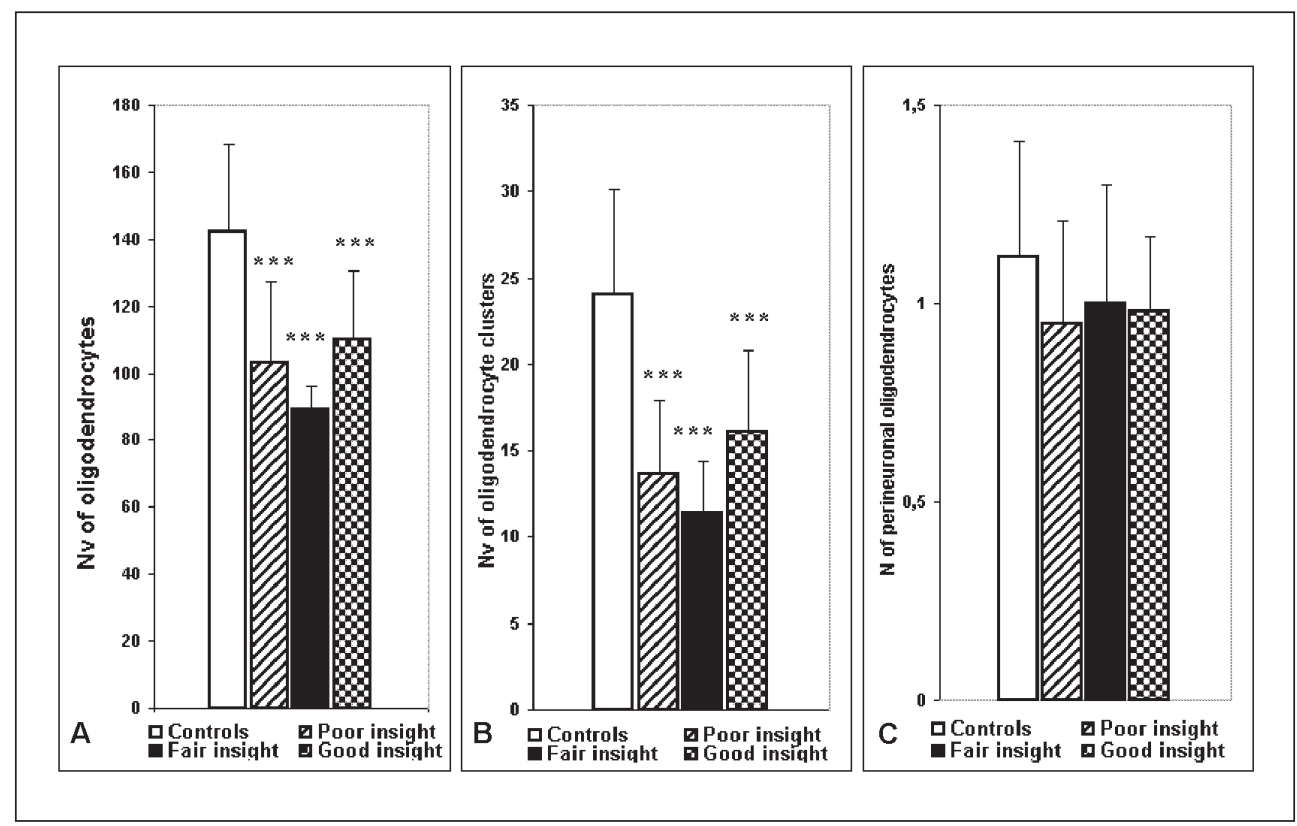

Figure 2. The numerical density of oligodendrocytes ( $\mathrm{Nv} \mathrm{Ol})(\mathrm{A})$, oligodendrocyte clusters (Nv OlC) (B) and the number of perineuronal oligodendrocytes $(\mathrm{N} \mathrm{PnOl})(\mathrm{C})$ in $\mathrm{BA} 40$ in the control group and in different insight subgroups. Data are given as mean \pm standard deviation. $\mathrm{p}$-values: $* * * \mathrm{p}<0.001$.

were associated with lack of insight. The results of the present study are in a good agreement with the data from the previous studies: in BA 39 the $\mathrm{Nv} \mathrm{Ol}$ and the $\mathrm{Nv}$ of $\mathrm{OlC}$ were decreased by $19 \%$, and the $\mathrm{N} \mathrm{PnOl}$ by $16 \%$ in the schizophrenia group as compared to the control group. Also, all these parameters were significantly lower in poor insight subgroup as compared to controls though there were no significant differences between the insight subgroups.

The parameters measured were not correlated with age, postmortem interval, refrigerator interval, brain weight, brain $\mathrm{pH}$, lifetime antipsychotics. A decrease in the mean values of the oligodendrocyte parameters was not due to the changes in laminar thickness, because Smiley et al. ${ }^{9}$ using the same IPL section collection as we used has not detected a significant changes in the laminar thickness and volume measurements, as well in neuronal size and density in BA 39/40 in schizophrenia.

Thus taken together these data provide evidence for the wide a spread layer deficit of oligodendrocytes in BA 39 in schizophrenia. Our data suggest that lack of insight in schizophrenia may be associated with the deficit of oligodendrocytes in grey matter of BA 39. These data are in agreement with the neuroimaging studies that the IPL activity was decreased in the schizophrenia patients ${ }^{3-5}$, correlated with cognitive insight in schizophrenia patients ${ }^{3}$, and the level of cognitive insight was correlated with the severity of psychotic symptoms in schizophrenia ${ }^{10}$.

The only one difference between the layers in BA 39 was the absence of hemispheric differences in layer 5 in contrast to layer 3. This difference is not surprising because the 
axons from layer 3 neurons give rise to the hemispheric connections in contrast to layer 5 neurons that project to different other cortical and sub-cortical brain structures.

The prominent deficit of $\mathrm{OlC}$ was also found in layer 3 in BA 40 in schizophrenia $(30 \%)^{7}$. The present study demonstrated the reduced $\mathrm{Nv} \mathrm{Ol} \mathrm{(-28 \% )} \mathrm{and} \mathrm{Nv}$ OlC (-42\%) in layer 5 of BA 40 in the schizophrenia group when compared to the control group. The $\mathrm{N}$ PnOl tended to decrease $(-14 \%, \mathrm{p}=0.06)$ in schizophrenia as compared to controls. Decreased N OlC in the grey matter of the IPL in schizophrenia is in line with the data of Hof et al. ${ }^{11}$ of a less clustered oligodendrocyte arrangement in the prefrontal cortex in schizophrenia compared to healthy controls.

There were no hemispheric differences in either layer 3 or layer 5 of BA 40 in both groups studied in contrast to BA $39^{6-8}$. Also in BA 40 in contrast to BA 39 each of three insights subgroups differed from the control group in the $\mathrm{Nv} \mathrm{Ol}$ and in the $\mathrm{Nv} \mathrm{OlC}$. These data suggest that in BA 40 the decreased values of these parameters are not related to insight. Thus, we showed some specificity in the deficit of $\mathrm{Nv} \mathrm{Ol}$ and $\mathrm{Nv} \mathrm{OlC}$ in the supramarginal gyrus (BA 40) as compared to the angular gyrus (BA 39). In layer 3 but not in layer 5 of BA 40 the $\mathrm{N}$ $\mathrm{PnOl}$ was significantly lower $(-17 \%)$ in the schizophrenia group as compared to the control group $^{8}$. Besides, the cytoarchitectonic areas of the human IPL are highly variable ${ }^{12}$ thus supporting the difference between BA 39 and BA 40 found in our study.

Recent experimental data demonstrated that cell clusters in adult rodent and primate brain contain oligodendrocyte progenitors at different stages of maturation ${ }^{13,14}$. Adult mammalian brain contains ubiquitous population of glial progenitors that develop into mature oligodendrocytes ${ }^{15}$. Besides, cell cycle abnormalities and incomplete differentiation of oligodendrocytes have been reported in schiz- ophrenia $^{16-18}$. The data suggest that $\mathrm{OlC}$ in the human IPL may represent sites of oligodendrocyte precursors proliferation and differentiation, and the reduced $\mathrm{Nv} \mathrm{OlC}$ in schizophrenia may be associated with the reduced proliferation of oligodendrocyte progenitors. We hypothesize that renewing of oligodendrocytes might be altered in schizophrenia.

Oligodendrocyte abnormalities may be involved in disturbance of neuronal connectivity in schizophrenia and may also contribute to the various clinical symptoms of schizophrenia including negative symptoms and impaired insight into their disorder ${ }^{19,20}$. The deficit of the Nv Ol, Nv OlC and the N $\mathrm{PnOl}$ in the IPL is associated with impaired insight and may play a key role in the pathophysiology of schizophrenia.

\section{Authors' contributions}

Dr. Uranova designed the study, analyzed data and wrote the manuscript. Dr. Vostrikov carried out data collection, contributed in interpretation and preparation the manuscript. Dr. Kolomeets contributed in data collection, interpretation and preparation of the manuscript. All authors have approved the final manuscript.

\section{Acknowledgments}

The authors would like to thank the Stanley Medical Research Institute for support this work. Postmortem brain sections were donated by Dr. M.J Webster from the Stanley 'Parietal Collection'. We express our gratitude to Dr. E. Fuller Torrey for examination of clinical records. 


\section{Conflict of interest}

All authors declare that they have no conflicts of interest.

\section{Funding source}

This study was supported by the Stanley Medical Research Institute. Grant number: 07R-1787.

\section{References}

1. Torrey EF. Schizophrenia and the inferior parietal lobule. Schizophr Res. 2007; 97(1-3): 215-25.

2. Cooke MA, Fannon D, Kuipers E, Peters E, Williams SC, Kumari V Neurological basis of poor insight in psychosis: A voxel-based MRI study. Schizophr Res. 2008; 103(1-3): 40-51.

3. Lee JS, Chun JW, Lee SH, Kim E, Lee SK, Kim JJ. Altered neural basis of the reality processing and its relation to cognitive insight in schizophrenia. PLoS One. 2015; 10(3): e0120478.

4. Kim GW, Chung YC, Yang JC, Chung GH, Park TJ, Jeong GW. Neuroanatomical mechanism on the effect of distraction in working memory maintenance in patients with schizophrenia. J Neuropsychiatry Clin Neurosci. 2015; 27(1): e1-9.

5. Zhou Y, Ma X, Wang D, Qin W, Zhu J, Zhuo C, Yu C. The selective impairment of resting-state functional connectivity of the lateral subregion of the frontal pole in schizophrenia. PLoS One. 2015; 10(3): e0119176.

6. V.M. Vostrikov, N. S. Kolomeets, N.A. Uranova. Reduced oligodendroglial density in neocortex and lack of insight in schizophrenia. Eur J Psychiat. 2013; 2: 111-21.

7. N.S. Kolomeets, V.M. Vostrikov, N.A. Uranova. Abnormalities in oligodendrocyte clusters in the inferior parietal cortex in schizophrenia are associated with insight. Eur J Psychiat. 2013; 4: 248-58.

8. V.M. Vostrikov, N.S. Kolomeets and N.A.Uranova Deficit of perineuronal oligodendrocytes in the inferior parietal cortex in schizophrenia is associated with insight schizophrenia. Eur J Psychiat. 2014; 28, 2: 114-23.

9. Smiley JF, Konnova K, Bleiwas C. Cortical thickness, neuron density and size in the inferior parietal lobe in schizophrenia. Schizophr Res. 2012; 136(1-3): 43-50.
10. Bora E, Erkan A, Kayahan B, Veznedaroglu B. Cognitive insight and acute psychosis in schizophrenia. Psychiatry Clin Neurosci. 2007; 61(6): 634-9.

11. Hof PR, Haroutunian V, Friedrich VL, Byne W, Buitron C, Perl DP, et al. Loss and altered spatial distribution of oligodendrocytes in the superior frontal gyrus in schizophrenia. Biol Psychiatry. 2003; 53(12): 1075-85.

12.Caspers S, Eickhoff SB, Geyer S, Scheperjans F, Mohlberg H, Zilles K, et al. The human inferior parietal lobule in stereotaxic space. Brain Struct Funct. 2008; 212: 481-95.

13. Nishiyama A, Komitova M, Suzuki R, Zhu X. Polydendrocytes (NG2 cells): Multifunctional cells with lineage plasticity. Nat Rev Neurosci. 2009;10(1): 9-22.

14. Chang A, Nishiyama A, Peterson J, Prineas J, Trapp BD. NG2-positive oligodendrocyte progenitor cells in adult human brain and multiple sclerosis lesions. J Neurosci. 2000; 20(17): 6404-12.

15. Radu A, Hristescu G, Katsel P, Haroutunian V, Davis KL. Microarray database mining and cell differentiation defects in schizophrenia. Adv Exp Med Biol. 2011; 696: 67-74.

16. Geha S, Pallud J, Junier MP, Devaux B, Leonard N, Chassoux F, et al. NG2+/Olig2+ cells are the major cyclerelated cell population of the adult human normal brain. Brain Pathol. 2010; 20(2): 399-411.

17. Wilson HC, Scolding NJ, Raine CS. Co-expression of PDGF alpha receptor and NG2 by oligodendrocyte precursors in human CNS and multiple sclerosis lesions. J Neuroimmunol. 2006; 176(1-2):162-73.

18. Katsel P, Davis KL, Li C, Tan W, Greenstein E, Kleiner Hoffman LB, et al. Abnormal indices of cell cycle activity in schizophrenia and their potential association with oligodendrocytes. Neuropsychopharmacology. 2008; 33(12): 2993-3009.

19. Antonius D, Prudent V, Rebani Y, D’Angelo D, Ar-dekani BA, Malaspina D, et al. White matter integrity and lack of insight in schizophrenia and schizoaffective disorder. Schizophr Res. 2011; 128(1-3): 76-82.

20. Larøi F, Fannemel M, Rønneberg U, Flekkøy K, Opjordsmoen S, Dullerud R. Unawareness of illness in chronic schizophrenia and its relationship to structural brain measures and neuropsychological tests. Psychiatr Res. 2000; 100: 49-58.

\footnotetext{
* Corresponding author:

Natalya A. Uranova

Laboratory of Clinical Neuropathology

Mental Health Research Center

Zagorodnoe shosse 2, 117152

Moscow, Russia

Tel. +7-495-952-87-30

Fax: +7-495-952-89-40

E-mail: uranovan@mail.ru
} 